\title{
Editorial
}

Psychopathology

\section{Delusions in Major Depressive Disorder: Recommendations for the DSM-V}

\author{
Mario Maj \\ Department of Psychiatry, University of Naples SUN, Naples, Italy
}

Delusions are a common symptom in major depressive disorder, occurring in about $25 \%$ of hospitalized patients and almost $15 \%$ of cases in the community [1, 2]. The phenomenology and prognostic significance of delusions in depressed patients are currently being discussed again within the context of the work on the DSM-V [3-6].

According to the DSM-IV [7], depressive thoughts, in order to be classified as delusions, must fulfill two criteria: (a) the judgment has to be so extreme as to defy credibility (i.e. the belief has to be of 'delusional proportions'), and (b) the patient must be unable to acknowledge the possibility that the belief may not be true (i.e. the belief has to be maintained with 'delusional intensity'). However, in clinical practice, there may be problems in applying these criteria. For instance, the delusional intensity of a belief may fluctuate during a depressive episode: some clinicians and researchers will classify the belief as a delusion even if the delusional intensity is present only for most of the time during the depressive episode, whereas others will require that the delusional intensity be consistently present throughout the depressive episode. Moreover, it happens that a belief fulfills one of the two criteria above but not the other: it is of delusional proportions but not maintained with delusional intensity, or vice versa. In these cases, clinicians and researchers may reach different conclusions about whether or not the patient has a delusion.

It is also useful to emphasize that, in the assessment of the presence of delusions in a depressed patient, both false negatives and false positives may occur. The presence of the delusions may be actively concealed by the patient (for instance, by a mother who is afraid that she will lose her children if she reveals her delusions), or the patient may be unable to fully recognize or report the presence of delusions because of perplexity, psychic retardation or agitation. On the other hand, the expressions sometimes used by patients when talking about their depressive feelings may be misleading, and some severely depressed patients may endorse the strongest terms used in structured interviews even if these terms do not apply to their particular case: this may generate false positives.

Delusions are not included in the DSM-IV criteria for a major depressive episode, but their presence can be recorded using the specifier 'severe with psychotic features', with the possibility to further specify whether the delusions are 'mood congruent' or 'mood incongruent' [7]. Thus, the DSM-IV seems to make two assumptions: (a) that every delusional depression will also be severe (i.e. characterized by the presence of several symptoms in excess to those required to make the diagnosis, and by a marked interference with social functioning), and (b) that, in an individual depressive episode, delusions will either be all congruent or all incongruent with depressed mood. In fact, it is not possible to record the concomitant presence of both mood-congruent and mood-incongruent delusions. However, some preliminary research evidence seems to question these two assumptions. One study reported that delusions can also be present in de-

\begin{tabular}{ll}
\hline KARGER & ( 2007 S. Karger AG, Basel \\
0254-4962/08/0411-0001\$24.50/0 \\
$\begin{array}{l}\text { Fax +41 61 306 1234 } \\
\text { www.karger.com }\end{array}$ & $\begin{array}{l}\text { Accessible online at: } \\
\text { www.karger.com/psp }\end{array}$
\end{tabular}

Mario Maj

Department of Psychiatry, University of Naples SUN

Largo Madonna delle Grazie

IT-80138 Naples (Italy)

Tel. +39 081566 6501, Fax +39081566 6523, E-Mail majmario@tin.it 
pressive episodes that are classified as either mild or moderate [8]. Several studies found that mood-congruent and mood-incongruent delusions may coexist in the same depressive episode [9-11]. In addition to this, it has been observed that the congruence of delusions with mood is sometimes difficult to evaluate, especially in persecutory delusions [12], although so far no study has reported the frequency of this problem.

Finally, the DSM-IV assumes that the presence of delusions and their mood-incongruent nature have significant prognostic implications. In fact, it states that 'suicide risk is especially high for individuals with psychotic features' and that mood-incongruent psychotic features 'are associated with a poorer prognosis' [7]. However, at present there is mixed evidence concerning the prognostic significance of these aspects [13-15], and further research is needed in order to clarify whether these statements should be kept in the DSM-V or modified in some way.

We have recently completed a 10 -year prospective follow-up study in a large patient population, aiming to systematically explore the phenomenology and prognostic significance of delusions in major depressive disorder and, on the basis of the results, to provide some recommendations for the relevant chapter of the DSM-V [16].

Out of the 452 patients fulfilling DSM-III criteria for major depression without a previous history of a manic or hypomanic episode, 89 (19.7\%) had a score of 3 on at least one of the Comprehensive Psychopathological Rating Scale items 'pessimistic thoughts', 'hypochondriasis', 'ideas of persecution' and 'feeling controlled' (i.e. at least one of their beliefs fulfilled both DSM-III prerequisites for delusions).

Of the 89 delusional patients, $65.2 \%$ had mood-congruent delusions only; $17.6 \%$ had mood-incongruent delusions only; $9.0 \%$ had both mood-congruent and moodincongruent delusions; $5.6 \%$ had both mood-congruent delusions and delusions with uncertain mood congruence, and 5.6\% had only delusions with uncertain mood congruence. All delusions with uncertain mood congruence were persecutory. In one third of the persecutory delusions, mood incongruence was uncertain.

Of the 452 evaluated patients, 123 (27.2\%) had no delusions but at least one sustained preoccupation (i.e. a score of 2 on at least one of the above-mentioned Comprehensive Psychopathological Rating Scale items). In 19.5\% of them, the score was 2 because the belief fulfilled only one of the DSM-III prerequisites for delusions (i.e. it was of 'delusional proportions' but not maintained with 'delusional intensity', or vice versa). In $13.8 \%$ of them, the belief was maintained with delusional intensity for only part of the episode, and thus we considered the criterion of delusional intensity as not fulfilled.

So, our data confirm that delusions and sustained preoccupations are common in major depressive disorder. They may coexist in a depressive episode, and the differentiation between them may be difficult. In the DSM-V, it would be advisable to provide a more detailed definition of what constitutes a delusion in a major depressive episode, including several examples to guide the clinician and the researcher. It should be clear that the delusional intensity of the belief has to be maintained consistently throughout the episode (i.e. at no point during the course of the episode should the patient have acknowledged that the belief might be unreasonable).

Moreover, in the DSM-V, it will be advisable to allow recording mood-congruent and mood-incongruent psychotic features at the same time in an individual patient, or to use the expression 'with predominant' mood-congruent or mood-incongruent psychotic features. It would be also useful to provide more detailed criteria for the evaluation of the mood congruence of delusions, with several clinical examples.

In our patients with delusions, the index episode was more likely to be 'severe' than in patients with sustained preoccupations but without delusions and in those without either delusions or sustained preoccupations. However, the index episode was either mild or moderate in $23.6 \%$ of patients with delusions. Therefore, in the DSM$\mathrm{V}$, it will be advisable to provide distinct specifiers for 'severity' and 'psychosis'.

When compared with patients without either delusions or sustained preoccupations, patients with delusions were more likely to show psychomotor agitation and perplexity and to receive antipsychotic medication during the index episode. Moreover, a multiple linear regression analysis showed that a longer time to syndromal recovery from the index episode was significantly associated with the presence of delusions. This evidence - that the presence of delusions has significant therapeutic and short-term prognostic implications - supports the clinical usefulness of the psychotic subtype in the diagnosis of major depressive disorder.

The percentage of time spent in a depressive episode during the prospective observation period was significantly longer in patients with delusions in their index episode, but not in those with sustained preoccupations only. However, at the 10-year follow-up interview, no variable, including the presence of delusions in the index episode, was found to have a significant effect on the global score of the Strauss-Carpenter Outcome Scale. 
This may indicate that the prognostic significance of delusions in major depression tends to become weaker over time.

Patients with mood-incongruent delusions did not differ significantly from those with mood-congruent delusions only for any demographic, historical or index episode variable, or with respect to outcome measures. This finding does not support the DSM-IV assumption that mood-incongruent psychotic features in major depressive disorder are associated with a poorer prognosis. The percentage of patients in our sample who had at least one 'bizarre' delusion according to the DSM-IV definition was too low (2.4\%) to allow an assessment of the prognostic significance of such delusions in major depressive disorder.

In summary, the empirical evidence provided by our study supports the usefulness of the 'psychotic' specifier in the diagnosis of major depressive disorder, since the presence of delusions in a major depressive episode was found to have significant therapeutic and short-term prognostic implications. However, the study indicates that the boundary between delusions and non-delusional sustained preoccupations in major depressive disorder may be somewhat blurred, and that some of the DSM-IV assumptions concerning psychotic depression (i.e. the assumption that this depression is always 'severe', that in an individual patient delusions will either be all congruent or all incongruent with depressed mood, and that moodincongruent delusions are associated with a poorer prognosis) may not be warranted.

Our findings suggest that the new edition of the DSM should be more explicit in clarifying what constitutes a delusion in a major depressive episode, with several clinical examples to guide the clinician and the researcher. It may be useful to specify that a belief should be classified as delusional only if consistently maintained with 'delusional intensity' throughout the depressive episode. More detailed criteria and clinical examples should also be provided for the evaluation of the mood congruence of delusions (especially of delusions of persecution) in depressed patients. It should be allowed to record mood-congruent and mood-incongruent psychotic features at the same time in an individual patient. Distinct specifiers should be provided for 'severity' and 'psychosis'. All definitions and clinical examples should be included in the text of the section on major depressive disorder (rather than in the final 'glossary of technical terms').

A clearer and more detailed phenomenological characterization of delusions in major depressive disorder is likely to increase their utility in predicting outcome and treatment response.

\section{References}

$>1$ Coryell W, Pfohl B, Zimmerman M: The clinical and neuroendocrine features of psychotic depression. J Nerv Ment Dis 1984;172: 521-528.

- Johnson J, Horwath E, Weissman MM: The validity of major depression with psychotic features based on a community study. Arch Gen Psychiatry 1991;48:1075-1081.

3 Schatzberg AF: Psychotic major depression. Presented at the DSM Research Planning Conference on Deconstructing Psychosis, Arlington, VA, February 15-17, 2006.

4 Maj M: Psychotic major depression: discussion. Presented at the DSM Research Planning Conference on Deconstructing Psychosis, Arlington, VA, February 15-17, 2006.

5 Maj M: Delusions in major depressive disorder: recommendations for DSM-V. Presented at the 160th Annual Meeting of the American Psychiatric Association, San Diego, CA, May 19-24, 2007.
6 Keller J, Schatzberg AF, Maj M: Current issues in classification of psychotic major depression. Schizophr Bull 2007;33:877-885.

7 American Psychiatric Association: Diagnostic and Statistical Manual of Mental Disorders, ed 4. Washington, American Psychiatric Association, 1994.

-8 Ohayon MM, Schatzberg AF: Prevalence of depressive episodes with psychotic features in the general population. Am J Psychiatry 2002;159:1855-1861.

-9 Black DW, Nasrallah A: Hallucinations and delusions in 1,715 patients with unipolar and bipolar affective disorders. Psychopathology 1989;22:28-34.

10 Burch EA Jr, Anton RF, Carson WH: Mood congruent and incongruent psychotic depressions: are they the same? J Affect Disord 1994;31:275-280.

11 Fennig S, Bromet EJ, Tanenberg-Karant M, et al: Mood-congruent versus mood-incongruent psychotic symptoms in first-admission patients with affective disorder. J Affect Disord 1996;37:23-29.
12 Brown RP: Delusional depression; in Mann JJ (ed): Phenomenology of Depressive Illness. New York, Human Sciences Press, 1988, pp 158-175.

13 Roose SP, Glassman AH, Walsh T, et al: Depression, delusions, and suicide. Am J Psychiatry 1983;140:1159-1162.

14 Black DW, Winokur G, Nasrallah A: Effect of psychosis on suicide risk in 1,593 patients with unipolar and bipolar affective disorders. Am J Psychiatry 1988;145:849-852.

15 Wolfersdorf M, Keller F, Steiner B, Hole G: Delusional depression and suicide. Acta Psychiatr Scand 1987;76:359-363.

16 Maj M, Pirozzi R, Magliano L, et al: Phenomenology and prognostic significance of delusions in major depressive disorder: a 10-year prospective follow-up study. J Clin Psychiatry, in press. 